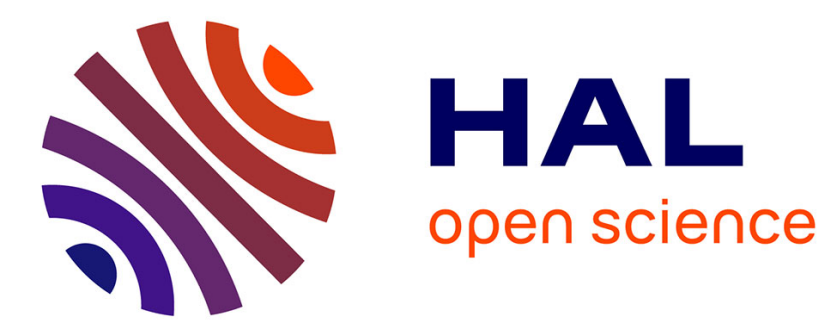

\title{
Study of a singular equation set in the half-space
} Chérif Amrouche, Fabien Dahoumane, Guy Vallet

\section{To cite this version:}

Chérif Amrouche, Fabien Dahoumane, Guy Vallet. Study of a singular equation set in the half-space. 2010. hal-00629120

\section{HAL Id: hal-00629120 https://hal.science/hal-00629120}

Preprint submitted on 5 Oct 2011

HAL is a multi-disciplinary open access archive for the deposit and dissemination of scientific research documents, whether they are published or not. The documents may come from teaching and research institutions in France or abroad, or from public or private research centers.
L'archive ouverte pluridisciplinaire HAL, est destinée au dépôt et à la diffusion de documents scientifiques de niveau recherche, publiés ou non, émanant des établissements d'enseignement et de recherche français ou étrangers, des laboratoires publics ou privés. 


\title{
STUDY OF A SINGULAR EQUATION SET IN THE HALF-SPACE
}

\author{
C. Amrouche, F. Dahoumane, G. Vallet
}

July 16, 2010

\author{
Laboratoire de Mathématiques Appliquées UMR-CNRS $N^{\circ} 5142$ \\ IPRA BP 1155 \\ 64013 Pau cedex \\ cherif.amrouche@univ-pau.fr,fabien.dahoumane@univ-pau.fr,guy.vallet@univ-pau.fr
}

Abstract. This work is dedicated to the resolution of a singular equation set in the half-space, with a diffusion coefficient that blows up on the boundary. More precisely, for a datum $g: \mathbb{R}_{+}^{3} \rightarrow \mathbb{R}$, our problem consists in seeking $u: \mathbb{R}_{+}^{3} \rightarrow \mathbb{R}$ formally solution to:

$$
-\operatorname{div}\left(\frac{1}{x_{3}} \nabla u\right)=g \text { in } \mathbb{R}_{+}^{3}, \quad u=0 \text { on } \Gamma=\mathbb{R}^{2} \times\{0\} .
$$

We give existence and uniqueness results of weak and strong solutions in suitable weighted spaces, where the weight depends on $x_{3}$.

KEY wORDS. Elliptic equation, singular equation, half-space, weighted spaces, weak solutions, strong solutions, Hardy's inequality.

AMS subject ClAssifications. 35Q30, 35B40, 76D05, 34C35

\section{Introduction}

This work is devoted to the study of a singular elliptic equation set in the half-space $\mathbb{R}_{+}^{3}$. More precisely, for a datum $g: \mathbb{R}_{+}^{3} \rightarrow \mathbb{R}$, our problem consists in looking for $u: \mathbb{R}_{+}^{3} \rightarrow \mathbb{R}$ formally solution to:

$$
-\operatorname{div}\left(\frac{1}{x_{3}} \nabla u\right)=g \text { in } \mathbb{R}_{+}^{3}, \quad u=0 \text { on } \Gamma=\mathbb{R}^{2} \times\{0\} .
$$

To understand the origins of this equation, we turn to the work of Bresch, GuillénGonzález and Lemoine in [7], who study an analogous equation set in regular bounded domain $\omega \subset \mathbb{R}^{2}$. Given $h: \omega \rightarrow \mathbb{R}$ and $g: \omega \rightarrow \mathbb{R}$, the authors are looking for $\psi: \omega \rightarrow \mathbb{R}$ formally solution to:

$$
-\operatorname{div}\left(\frac{1}{h} \nabla \Psi\right)=g \text { in } \omega, \quad \Psi=0 \text { on } \partial \omega .
$$

Basically, $h$ is a positive Lispichtz-continuous mapping, behaving as the distance function $\delta\left(x^{\prime}\right)=\operatorname{dist}\left(x^{\prime}, \partial \omega\right), x^{\prime} \in \omega$, in a neighbourhood of $\partial \omega$. 
The authors in [7] explain how such singular equation (2) naturally appears in the study of models issued from oceanography, under a hydrostatic pressure assumption and set in domains with vanishing depth. It is the case for example of the planetary geostrophic equation [9], the vertical-geostrophic equations [8], and the hydrostatic Stokes or Navier-Stokes equations $[1,3,5,11,15]$. Before stating their main result, we define the space

$$
H(\omega)=\left\{\Psi \in L^{2}(\omega) / h^{-1 / 2} \nabla \psi \in L^{2}(\omega)^{2}, \Psi=0 \text { on } \partial \omega\right\}
$$

endowed with the norm $\|\Psi\|_{H(\omega)}=\left\|h^{-1 / 2} \nabla \Psi\right\|_{L^{2}(\omega)^{2}}$.

Theorem 1.1. Let $g$ be such that $\delta h^{1 / 2} g \in L^{2}(\omega)$. There exists a unique solution $\Psi$ of (2) such that $\Psi \in H(\omega)$ and:

$$
\|\Psi\|_{H(\omega)} \leqslant C\left\|\delta h^{1 / 2} g\right\|_{L^{2}(\omega)} .
$$

Moreover, if $h^{1 / 2} g \in L^{2}(\omega)$, then:

$$
h^{1 / 2} \nabla\left(\frac{1}{h} \nabla \Psi\right) \in L^{2}(\omega)^{4}, \quad\left\|h^{1 / 2} \nabla\left(\frac{1}{h} \nabla \Psi\right)\right\|_{L^{2}(\omega)^{4}} \leqslant C\left\|h^{1 / 2} g\right\|_{L^{2}(\omega)} .
$$

To our knowledge, no theoretical study was made in the case of the half-space $\mathbb{R}_{+}^{2}$, however usefull to obtain a good comprehension of the general case of a bounded domain. This paper is therefore devoted to the study of such a case, see (1). We present the case of the dimension 3 only for technical reasons, explained later.

On the one hand, our work enables to achieve the following result similar to Theorem 1.1 , in a context of weighted spaces, where the weight depends on $x_{3}$. Beforehand, we introduce prematurely the space $W_{-\frac{1}{2}, x_{3}}^{1,2}\left(\mathbb{R}_{+}^{3}\right)$, see $(11)$, endowed with the norm given by (12).

Theorem 1.2. Let $g \in \mathcal{D}^{\prime}\left(\mathbb{R}_{+}^{3}\right)$ such that $x_{3}^{3 / 2} g \in L^{2}\left(\mathbb{R}_{+}^{3}\right)$. Then, there exists a unique solution $u \in W_{-\frac{1}{2}, x_{3}}^{1,2}\left(\mathbb{R}_{+}^{3}\right)$ of (1). Moreover, there is a positive constant $C$ such that:

$$
\|u\|_{W_{-\frac{1}{2}, x_{3}}^{1,2}\left(\mathbb{R}_{+}^{3}\right)} \leqslant C\left\|x_{3}^{3 / 2} g\right\|_{L^{2}\left(\mathbb{R}_{+}^{3}\right)} .
$$

If in addition, $\sqrt{x_{3}} g \in L^{2}\left(\mathbb{R}_{+}^{3}\right)$, then

$$
\sqrt{x_{3}} \nabla\left(\frac{1}{x_{3}} \nabla u\right) \in L^{2}\left(\mathbb{R}_{+}^{3}\right)^{9}, \quad\left\|\sqrt{x_{3}} \nabla\left(\frac{1}{x_{3}} \nabla u\right)\right\|_{L^{2}\left(\mathbb{R}_{+}^{3}\right)^{9}} \leqslant C\left\|\sqrt{x_{3}} g\right\|_{L^{2}\left(\mathbb{R}_{+}^{3}\right)} .
$$

On the other hand, we consider a larger class of data $g$ in suitable weighted spaces, and give results of existence and uniqueness of weak and strong solutions to (1), that include Theorem 1.2.

Note that the ideas contained in this work, can be adapted to the $\mathbb{R}_{+}^{N}$-case where $N \geqslant 3$. In the $\mathbb{R}_{+}^{2}$-case, it is necessary to introduce an additional logarithmic weight, which complicates a bit more the presentation of the results. This is why we limit our study to the case of the dimension 3 .

An outline of this paper is as follows. In Section 2, we set the functional framework, by introducting two kinds of weighted spaces. Firstly, we give an overview of the weighted spaces $W_{\alpha}^{1,2}\left(\mathbb{R}_{+}^{3}\right)$, see (4), encountered in works concerning for example Laplace equation studied in $\mathbb{R}_{+}^{3}$. Secondly, we will build another family of weighted spaces $W_{\alpha, x_{3}}^{1,2}\left(\mathbb{R}_{+}^{3}\right)$, see (11), where the weight depends on $x_{3}$, and especially adapted to the study of (1). Following the work of Grisvard [12], we will define a trace operator, 
give density results and generalized Hardy's inequalities. We will finish this section with a comparaison between these two families of weighted spaces, proving continuous and dense embeddings between them.

In Section 3, we study the existence and uniqueness of weak solutions to (1), by considering a large class of data $g$. More precisely, we prove in Theorem 3.2 the existence and uniqueness of a weak solution to (1) in $W_{\alpha, x_{3}}^{1,2}\left(\mathbb{R}_{+}^{3}\right)$, while in Theorem 3.4 , existence and uniqueness of a weak solution to $(1)$ is obtained in $W_{\alpha}^{1,2}\left(\mathbb{R}_{+}^{3}\right)$.

The last section of this paper is devoted to the study of strong solutions to (1), completing the theoretical study of Problem (1). More precisely, we give two situations where the weak solution of Theorem 3.2 is in fact a strong solution, see Theorem 4.1 and Theorem 4.5.

\section{Functional framework}

This first section is aimed to set the functional framework adpated to the study of Problem (1). In particular, we define and study here the appropriate space for weak solutions to $(1)$.

Let us make the following observation. Let us consider a datum $g$ such that $x_{3} g \in$ $L^{2}\left(\mathbb{R}_{+}^{3}\right)$. Formally, let us multiply (1) by $x_{3} u$ and integrate over $\mathbb{R}_{+}^{3}$. Then, using Green's formula and taking into account the boundary condition over $u$, we deduce the following energy equality:

$$
\int_{\mathbb{R}_{+}^{3}}|\nabla u|^{2} d x+\frac{1}{2} \int_{\mathbb{R}_{+}^{3}}\left|\frac{u}{x_{3}}\right|^{2} d x=\int_{\mathbb{R}_{+}^{3}} x_{3} g u d x
$$

Therefore, we are naturally lead to look for weak solutions $u$ such that:

$$
\nabla u \in L^{2}\left(\mathbb{R}_{+}^{3}\right)^{3}, \quad \frac{u}{x_{3}} \in L^{2}\left(\mathbb{R}_{+}^{3}\right), \quad u=0 \text { on } \Gamma .
$$

This first situation is considered in Proposition 3.1, where we establish the existence and uniqueness of such a weak solution. Then, since $\left|x_{3}\right| \leqslant\left(1+|x|^{2}\right)^{1 / 2}$, any weak solution satisfying $(3)$ is in fact in the weighted Sobolev space $W_{0}^{1,2}\left(\mathbb{R}_{+}^{3}\right)$, where:

$$
W_{0}^{1,2}\left(\mathbb{R}_{+}^{3}\right)=\left\{u \in \mathcal{D}^{\prime}\left(\mathbb{R}_{+}^{3}\right) / \frac{u}{\left(1+|x|^{2}\right)^{1 / 2}} \in L^{2}\left(\mathbb{R}_{+}^{3}\right) \text { and } \nabla u \in L^{2}\left(\mathbb{R}_{+}^{3}\right)^{3}\right\} .
$$

And therefore, the boundary condition $u=0$ on $\Gamma$ is meaningfull.

Let us recall properties of the weighted Sobolev spaces $W_{\alpha}^{1,2}\left(\mathbb{R}_{+}^{3}\right)$, usefull in the sequel, and study a new familly of weighted spaces, where the weight depends on $x_{3}$, see (11).

\subsection{An overview of the weighted Sobolev spaces $W_{\alpha}^{1,2}\left(\mathbb{R}_{+}^{3}\right)$}

In a general way, the spaces $W_{\alpha}^{1,2}\left(\mathbb{R}_{+}^{3}\right), \alpha \in \mathbb{R}$, have been introduced since they are adapted to the study of some second order elliptic equations set in $\mathbb{R}_{+}^{3}$, and more precisely, to the Laplace equation with inhomogeneous Dirichlet or Neuman boundary conditions. We refer here to the work of Amrouche and Nečasova [2], Boulmezaoud [6], or the one of Hanouzet [13].

Let $\alpha \in \mathbb{R}$ and set for any $x \in \mathbb{R}_{+}^{3}, \rho(x)=\sqrt{1+|x|^{2}}$, where $|x|$ denotes the euclidean norm of $x$. Then, we introduce the weighted Sobolev space:

$$
W_{\alpha}^{1,2}\left(\mathbb{R}_{+}^{3}\right)=\left\{u \in \mathcal{D}^{\prime}\left(\mathbb{R}_{+}^{3}\right) / \rho^{\alpha-1} u \in L^{2}\left(\mathbb{R}_{+}^{3}\right) \text { and } \rho^{\alpha} \nabla u \in L^{2}\left(\mathbb{R}_{+}^{3}\right)^{3}\right\},
$$


which is a Hilbert space for the norm:

$$
\|u\|_{W_{\alpha}^{1,2}\left(\mathbb{R}_{+}^{3}\right)}=\left(\left\|\rho^{\alpha-1} u\right\|_{L^{2}\left(\mathbb{R}_{+}^{3}\right)}^{2}+\left\|\rho^{\alpha} \nabla u\right\|_{L^{2}\left(\mathbb{R}_{+}^{3}\right)^{3}}^{2}\right)^{1 / 2} .
$$

The weight $\rho$ is chosen such that the space $W_{\alpha}^{1,2}\left(\mathbb{R}_{+}^{3}\right)$ satisfies two fundamental properties. On the one hand, $\mathcal{D}\left(\overline{\mathbb{R}_{+}^{3}}\right)$ is dense in $W_{\alpha}^{1,2}\left(\mathbb{R}_{+}^{3}\right)$ (see [13]). On the other hand, if $\alpha \neq-1 / 2$, any functions of $W_{\alpha}^{1,2}\left(\mathbb{R}_{+}^{3}\right)$ satisfies a Poincaré type inequality (see $[2]$ ).

As $\frac{1}{\rho}$ is bounded, note that for any $\alpha \geqslant \beta$, the following continuous embedding holds:

$$
W_{\alpha}^{1,2}\left(\mathbb{R}_{+}^{3}\right) \hookrightarrow W_{\beta}^{1,2}\left(\mathbb{R}_{+}^{3}\right),
$$

Then, any function $u$ in $W_{\alpha}^{1,2}\left(\mathbb{R}_{+}^{3}\right)$ has a trace on $\Gamma$, and $\left.u\right|_{\Gamma}$ belongs to the weighted space $W_{\alpha}^{1 / 2,2}(\Gamma)$. For more precision, we refer to [13].

Theorem 2.1. The mapping $\gamma: u \mapsto u\left(x^{\prime}, 0\right)$ defined on $\mathcal{D}\left(\overline{\mathbb{R}_{+}^{3}}\right)$, can be extended in a unique way to a linear and continuous mapping, still denoted by $\gamma$, from $W_{\alpha}^{1,2}\left(\mathbb{R}_{+}^{3}\right)$ into $W_{\alpha}^{1 / 2,2}(\Gamma)$.

The following short paragraph is dedicated to basic properties of the space $\stackrel{\circ}{\alpha}_{\alpha}^{1,2}\left(\mathbb{R}_{+}^{3}\right)$, the closure of $\mathcal{D}\left(\mathbb{R}_{+}^{3}\right)$ in $W_{\alpha}^{1,2}\left(\mathbb{R}_{+}^{3}\right)$. Firstly, when $\alpha \neq-1 / 2$ and according for example to [6], we have the following Hardy type inequality:

$$
\forall u \in \stackrel{\circ}{W}_{\alpha}^{1,2}\left(\mathbb{R}_{+}^{3}\right), \quad\left\|\rho^{\alpha-1} u\right\|_{L^{2}\left(\mathbb{R}_{+}^{3}\right)} \leqslant \mathcal{H}_{\alpha}\left\|\rho^{\alpha} \nabla u\right\|_{L^{2}\left(\mathbb{R}_{+}^{3}\right)^{3}},
$$

where $\mathcal{H}_{\alpha}$ is a positive constant, depending only on $\alpha$. And following for example the proof of Theorem I.2 of [13], we can choose $\mathcal{H}_{\alpha}=\frac{2}{(2 \alpha+1)}$ when $0 \leqslant \alpha \leqslant 1$, yielding finally:

$$
\forall u \in \stackrel{\circ}{W}_{\alpha}^{1,2}\left(\mathbb{R}_{+}^{3}\right), \quad\left\|\rho^{\alpha-1} u\right\|_{L^{2}\left(\mathbb{R}_{+}^{3}\right)} \leqslant \frac{2}{(2 \alpha+1)}\left\|\rho^{\alpha} \nabla u\right\|_{L^{2}\left(\mathbb{R}_{+}^{3}\right)^{3}} .
$$

As a consequence of (7) and still if $\alpha \neq-1 / 2$, the mapping $u \mapsto\left\|\rho^{\alpha} \nabla u\right\|_{L^{2}\left(\mathbb{R}_{+}^{3}\right)^{3}}$ defines a norm on $\stackrel{\circ}{\alpha}_{\alpha}^{1,2}\left(\mathbb{R}_{+}^{3}\right)$, equivalent to the one defined in (5). Secondly, it is also proved in [13] page 238, that for any $\alpha, \beta \in \mathbb{R}$ the mapping below is an isomorphism:

$$
T_{\rho, \beta}: u \in \stackrel{\circ}{W}_{\alpha}^{1,2}\left(\mathbb{R}_{+}^{3}\right) \mapsto \rho^{\beta} u \in \stackrel{\circ}{W}_{\alpha-\beta}^{1,2}\left(\mathbb{R}_{+}^{3}\right),
$$

and one has the estimate:

$$
\forall u \in \stackrel{\circ}{W}_{\alpha}^{1,2}\left(\mathbb{R}_{+}^{3}\right), \quad\left\|T_{\rho, \beta} u\right\|_{W_{\alpha-\beta}^{1,2}\left(\mathbb{R}_{+}^{3}\right)} \leqslant \sqrt{1+\beta^{2}}\|u\|_{W_{\alpha}^{1,2}\left(\mathbb{R}_{+}^{3}\right)} .
$$

Finally, and according to [13] page 258 Theorem II. 3, the space $\stackrel{\circ}{\alpha}_{\alpha}^{1,2}\left(\mathbb{R}_{+}^{3}\right)$ can be characterized as the kernel of $\gamma$ :

$$
\stackrel{\circ}{W}_{\alpha}^{1,2}\left(\mathbb{R}_{+}^{3}\right)=\left\{u \in W_{\alpha}^{1,2}\left(\mathbb{R}_{+}^{3}\right) / u=0 \text { on } \Gamma\right\} .
$$

We finish this paragraph by introducing the space of distributions $W_{-\alpha}^{-1,2}\left(\mathbb{R}_{+}^{3}\right)$ as the dual space of $\stackrel{\circ}{W}_{\alpha}^{1,2}\left(\mathbb{R}_{+}^{3}\right)$. 


\subsection{The spaces $W_{\alpha, x_{3}}^{1,2}\left(\mathbb{R}_{+}^{3}\right)$}

For $\alpha \in \mathbb{R}$, we consider the Hilbert space:

$$
W_{\alpha, x_{3}}^{1,2}\left(\mathbb{R}_{+}^{3}\right)=\left\{u \in \mathcal{D}^{\prime}\left(\mathbb{R}_{+}^{3}\right) / x_{3}^{\alpha-1} u \in L^{2}\left(\mathbb{R}_{+}^{3}\right), x_{3}^{\alpha} \nabla u \in L^{2}\left(\mathbb{R}_{+}^{3}\right)^{3}\right\},
$$

endowed with the norm:

$$
\|u\|_{W_{\alpha, x_{3}}^{1,2}\left(\mathbb{R}_{+}^{3}\right)}=\left(\left\|x_{3}^{\alpha-1} u\right\|_{L^{2}\left(\mathbb{R}_{+}^{3}\right)}^{2}+\left\|x_{3}^{\alpha} \nabla u\right\|_{L^{2}\left(\mathbb{R}_{+}^{3}\right)^{3}}^{2}\right)^{1 / 2} .
$$

In the early 1960s, a similar space has been studied by Grisvard, see [12]. In his work, Grisvard considers the case of functions $u$ satisfying:

$$
x_{3}^{\alpha} u \in L^{2}\left(\mathbb{R}_{+}^{3}\right), x_{3}^{\alpha} \nabla u \in L^{2}\left(\mathbb{R}_{+}^{3}\right)^{3} .
$$

He establishes crucial properties, such as the existence of a trace operator and density results. Following his ideas, we begin this section by establishing that when $\alpha<1 / 2$, any functions $u$ of $W_{\alpha, x_{3}}^{1,2}\left(\mathbb{R}_{+}^{3}\right)$ possesses a trace in $L^{2}\left(\mathbb{R}^{2}\right)$. The idea is to prove that any function $u$ of $W_{\alpha, x_{3}}^{1,2}\left(\mathbb{R}_{+}^{3}\right)$ is continuous in the vertical direction, ensuring therefore the existence of the expected trace.

Proposition 2.2. Let $\alpha<1 / 2$. The mapping $u \mapsto \gamma u$ defined by:

$$
\gamma u=\lim _{x_{3} \rightarrow 0} u\left(\cdot, x_{3}\right),
$$

is linear and continuous from $W_{\alpha, x_{3}}^{1,2}\left(\mathbb{R}_{+}^{3}\right)$ into $L^{2}\left(\mathbb{R}^{2}\right)$.

Proof. For convenience, we set $E=L^{2}\left(\mathbb{R}^{2}\right)$. Then, let us introduce the space $\left({ }^{1}\right)$ :

$$
F=\left\{v \in L_{(\alpha-1)}^{2}(0,+\infty ; E) / v^{\prime} \in L_{\alpha}^{2}(0,+\infty ; E)\right\},
$$

and note that $W_{\alpha, x_{3}}^{1,2}\left(\mathbb{R}_{+}^{3}\right)$ can be identified as a subspace of $F$, as a consequence of the theorem of Fubini. Therefore, if we prove that $F$ is continuously embedded in $C^{0}\left(\left[0,+\infty[; E)\right.\right.$, we are able to define $u(0)$ as well as $\gamma u \in E$ for any $u \in W_{\alpha, x_{3}}^{1,2}\left(\mathbb{R}_{+}^{3}\right)$. We focus now on establishing this embedding. It follows from the inequalities below, available for any $v \in F$ and $T>0$ :

$$
\begin{gathered}
\int_{0}^{T}\|v(t)\|_{E} d t \leqslant\left(\int_{0}^{+\infty}\|v(t)\|_{E}^{2} t^{2(\alpha-1)} d t\right)^{1 / 2}\left(\int_{0}^{T} t^{-2(\alpha-1)} d t\right)^{1 / 2}, \\
\int_{0}^{T}\left\|v^{\prime}(t)\right\|_{E} d t \leqslant\left(\int_{0}^{+\infty}\|v(t)\|_{E}^{2} t^{2 \alpha} d t\right)^{1 / 2}\left(\int_{0}^{T} t^{-2 \alpha} d t\right)^{1 / 2}
\end{gathered}
$$

that the mapping $v \mapsto\left(v, v^{\prime}\right)$ is continuous from $F$ into $L_{\text {loc }}^{1}\left(\left[0,+\infty[; E)^{2}\right.\right.$. As a consequence, one has proved that:

$$
F \hookrightarrow C^{0}([0,+\infty[; E),
$$

and the proof is finished.

We follow this section by establishing a density result, adapted from [12].

\footnotetext{
${ }^{1} L_{\alpha}^{2}(0,+\infty ; E)$ denotes the set of measurable functions $\left.v:\right] 0,+\infty[\rightarrow E$ such that:

$$
\int_{0}^{+\infty}\|v\|_{E}^{2} t^{2 \alpha} d t<\infty
$$
}


Theorem 2.3. For any $\alpha \in \mathbb{R}$, the space $\mathcal{D}\left(\mathbb{R}_{+}^{3}\right)$ is dense in $W_{\alpha, x_{3}}^{1,2}\left(\mathbb{R}_{+}^{3}\right)$. As a consequence, if $\alpha<1 / 2$ the functions of $W_{\alpha, x_{3}}^{1,2}\left(\mathbb{R}_{+}^{3}\right)$ has null trace on $\mathbb{R}^{2}$.

Proof. If we prove that $\mathcal{D}\left(\mathbb{R}_{+}^{3}\right)$ is dense in $W_{\alpha, x_{3}}^{1,2}\left(\mathbb{R}_{+}^{3}\right)$, then it follows from Proposition 2.2 that whenever the trace of $u$ is defined, it is necessarily equal to 0 .

Exceptionally in this proof, we denote by $\mathcal{E}^{\prime}\left(\mathbb{R}_{+}^{3}\right)$ the set of distributions with compact support in $\mathbb{R}_{+}^{3}$, and $\mathcal{E}^{\prime}\left(\overline{\mathbb{R}_{+}^{3}}\right)$ the set of distributions with compact support in $\overline{\mathbb{R}_{+}^{3}}$. The proof is then divided into three steps.

Step one. It is clear that $\mathcal{D}\left(\mathbb{R}_{+}^{3}\right)$ is dense in $W_{\alpha, x_{3}}^{1,2}\left(\mathbb{R}_{+}^{3}\right) \cap \mathcal{E}^{\prime}\left(\mathbb{R}_{+}^{3}\right)$, since any function in $W_{\alpha, x_{3}}^{1,2}\left(\mathbb{R}_{+}^{3}\right) \cap \mathcal{E}^{\prime}\left(\mathbb{R}_{+}^{3}\right)$ belongs to $H_{0}^{1}\left(\mathbb{R}_{+}^{3}\right) \cap \mathcal{E}^{\prime}\left(\mathbb{R}_{+}^{3}\right)$.

Step two. We prove that $W_{\alpha, x_{3}}^{1,2}\left(\mathbb{R}_{+}^{3}\right) \cap \mathcal{E}^{\prime}\left(\mathbb{R}_{+}^{3}\right)$ is dense in $W_{\alpha, x_{3}}^{1,2}\left(\mathbb{R}_{+}^{3}\right) \cap \mathcal{E}^{\prime}\left(\overline{\mathbb{R}_{+}^{3}}\right)$. Let $u$ in $W_{\alpha, x_{3}}^{1,2}\left(\mathbb{R}_{+}^{3}\right) \cap \mathcal{E}^{\prime}\left(\overline{\mathbb{R}_{+}^{3}}\right)$ and $\psi \in C^{1}([0,+\infty[)$, such that $0 \leqslant \psi \leqslant 1, \psi(t)=0$ if $t \leqslant 1, \psi(t)=1$ if $t \geqslant 2$. Then, let us define $\varphi_{\varepsilon}$ by:

$$
x \in \mathbb{R}_{+}^{3}, \quad \varphi_{\varepsilon}(x)=\psi\left(\frac{x_{3}}{\varepsilon}\right),
$$

and set $u_{\varepsilon}=u \varphi_{\varepsilon}$. One proves with no difficulty that $u_{\varepsilon} \in W_{\alpha, x_{3}}^{1,2}\left(\mathbb{R}_{+}^{3}\right) \cap \mathcal{E}^{\prime}\left(\mathbb{R}_{+}^{3}\right)$. Next, observe the following computations:

$$
\begin{aligned}
\int_{0}^{+\infty}\left\|u(t) \varphi_{\varepsilon}(t)-u(t)\right\|_{L^{2}\left(\mathbb{R}^{2}\right)}^{2} t^{2(\alpha-1)} d t & =\int_{0}^{2 \varepsilon}\left|\psi\left(\frac{t}{\varepsilon}\right)-1\right|\|u(t)\|_{L^{2}\left(\mathbb{R}^{2}\right)}^{2} t^{2(\alpha-1)} d t \\
& \leqslant \int_{0}^{2 \varepsilon}\|u(t)\|_{L^{2}\left(\mathbb{R}^{2}\right)}^{2} t^{2(\alpha-1)} d t .
\end{aligned}
$$

We deduce from Lebesgue's theorem that $u_{\varepsilon} \rightarrow u$ in $L_{\alpha-1}^{2}\left(0,+\infty, L^{2}\left(\mathbb{R}^{2}\right)\right)$, when $\varepsilon$ goes to 0 . It is also clear that $\varphi_{\varepsilon} \nabla u \rightarrow \nabla u$ in $L_{\alpha}^{2}\left(0,+\infty, L^{2}\left(\mathbb{R}^{2}\right)^{3}\right)$. Therefore, it remains to establish that $u \nabla \varphi_{\varepsilon} \rightarrow 0$ in $L_{\alpha}^{2}\left(0,+\infty, L^{2}\left(\mathbb{R}^{2}\right)^{3}\right)$ and this follows from:

$$
\begin{aligned}
\int_{0}^{+\infty}\left\|u(t) \nabla \varphi_{\varepsilon}(t)\right\|_{L^{2}\left(\mathbb{R}^{2}\right)^{3}}^{2} t^{2 \alpha} d t & =\int_{\varepsilon}^{2 \varepsilon}\left\|u(t) \psi^{\prime}\left(\frac{t}{\varepsilon}\right)\right\|_{L^{2}\left(\mathbb{R}^{2}\right)}^{2} \frac{t^{2 \alpha}}{\varepsilon^{2}} d t \\
& \leqslant 4 \int_{\varepsilon}^{2 \varepsilon}\|u(t)\|_{L^{2}\left(\mathbb{R}^{2}\right)}^{2} t^{2(\alpha-1)} d t
\end{aligned}
$$

Step three. We chek that $W_{\alpha, x_{3}}^{1,2}\left(\mathbb{R}_{+}^{3}\right) \cap \mathcal{E}^{\prime}\left(\overline{\mathbb{R}_{+}^{3}}\right)$ is dense in $W_{\alpha, x_{3}}^{1,2}\left(\mathbb{R}_{+}^{3}\right)$, using the usual process of truncation. Indeed, let $\varphi \in C^{1}\left(\overline{\mathbb{R}_{+}^{3}}\right)$ such that $0 \leqslant \varphi \leqslant 1, \varphi(x)=1$ if $|x| \leqslant 1, \varphi(x)=0$ if $|x| \geqslant 2$ and define $\varphi_{k}$ by:

$$
x \in \mathbb{R}_{+}^{3}, \quad \varphi_{k}(x)=\varphi\left(\frac{x}{k}\right) .
$$

Then for any $u \in W_{\alpha, x_{3}}^{1,2}\left(\mathbb{R}_{+}^{3}\right), u_{k}=u \varphi_{k}$ belongs to $W_{\alpha, x_{3}}^{1,2}\left(\mathbb{R}_{+}^{3}\right) \cap \mathcal{E}^{\prime}\left(\overline{\mathbb{R}_{+}^{3}}\right)$, and following Step two, we deduce that $u_{k}$ converges strongly to $u$ in $W_{\alpha, x_{3}}^{1,2}\left(\mathbb{R}_{+}^{3}\right)$.

Remark 2.4. According for example to [12], see Theorem 1.2 and Theorem 1.3, observe that for any $\alpha \neq 1 / 2$ the following Hardy's inequality holds:

$$
\forall u \in W_{\alpha, x}^{1,2}\left(\mathbb{R}_{+}^{3}\right), \quad\left\|x_{3}^{\alpha-1} u\right\|_{L^{2}\left(\mathbb{R}_{+}^{3}\right)} \leqslant \frac{2}{|2 \alpha-1|}\left\|x_{3}^{\alpha} \nabla u\right\|_{L^{2}\left(\mathbb{R}_{+}^{3}\right)^{3}} .
$$

Let us now introduce and characterize the dual space to $W_{\alpha, x_{3}}^{1,2}\left(\mathbb{R}_{+}^{3}\right)$.

Definition 2.5. Let us denote by $W_{-\alpha, x_{3}}^{-1,2}\left(\mathbb{R}_{+}^{3}\right)$ the dual space of $W_{\alpha, x_{3}}^{1,2}\left(\mathbb{R}_{+}^{3}\right)$. 
In the previous paragraph, we have proved that for any $\alpha \in \mathbb{R}$, the space $\mathcal{D}\left(\mathbb{R}_{+}^{3}\right)$ is dense in $W_{\alpha, x_{3}}^{1,2}\left(\mathbb{R}_{+}^{3}\right)$. As a consequence, the space $W_{-\alpha, x_{3}}^{-1,2}\left(\mathbb{R}_{+}^{3}\right)$ is a space of distributions, which can be characterized in the following way.

Proposition 2.6. Any distribution $g \in \mathcal{D}^{\prime}\left(\mathbb{R}_{+}^{3}\right)$ belongs to $W_{-\alpha, x_{3}}^{-1,2}\left(\mathbb{R}_{+}^{3}\right)$, if and only if there is $f_{0} \in L^{2}\left(\mathbb{R}_{+}^{3}\right)$ and $\boldsymbol{f} \in L^{2}\left(\mathbb{R}_{+}^{3}\right)^{3}$ such that:

$$
g=x_{3}^{\alpha-1} f_{0}+\operatorname{div}\left(x_{3}^{\alpha} \boldsymbol{f}\right),
$$

with the estimate below:

$$
\|g\|_{W_{-\alpha, x_{3}}^{-1,2}\left(\mathbb{R}_{+}^{3}\right)} \leqslant\left(\left\|f_{0}\right\|_{L^{2}\left(\mathbb{R}_{+}^{3}\right)}+\|\boldsymbol{f}\|_{L^{2}\left(\mathbb{R}_{+}^{3}\right)^{3}}\right) .
$$

Moreover, if $\alpha \neq 1 / 2$, one can take $f_{0}=0$.

Proof. Let $f_{0} \in L^{2}\left(\mathbb{R}_{+}^{3}\right)$ and $\boldsymbol{f} \in L^{2}\left(\mathbb{R}_{+}^{3}\right)^{3}$. Then, one has for any $\varphi \in \mathcal{D}\left(\mathbb{R}_{+}^{3}\right)$

$$
\begin{aligned}
\left|\left\langle x_{3}^{\alpha-1} f_{0}+\operatorname{div}\left(x_{3}^{\alpha} \boldsymbol{f}\right), \varphi\right\rangle\right| & =\left|\int_{\mathbb{R}_{+}^{3}} x_{3}^{\alpha-1} f_{0} \varphi d x-\int_{\mathbb{R}_{+}^{3}} x_{3}^{\alpha} \boldsymbol{f} \cdot \nabla \varphi d x\right| \\
& \leqslant\left\|f_{0}\right\|_{L^{2}\left(\mathbb{R}_{+}^{3}\right)}\left\|x_{3}^{\alpha-1} \varphi\right\|_{L^{2}\left(\mathbb{R}_{+}^{3}\right)}+\|\boldsymbol{f}\|_{L^{2}\left(\mathbb{R}_{+}^{3}\right)}\left\|x_{3}^{\alpha} \nabla \varphi\right\|_{L^{2}\left(\mathbb{R}_{+}^{3}\right)^{3}} \\
& \leqslant C\|\varphi\|_{W_{\alpha, x_{3}}^{1,2}\left(\mathbb{R}_{+}^{3}\right)},
\end{aligned}
$$

and by density we deduce that:

$$
x_{3}^{\alpha-1} f_{0}+\operatorname{div}\left(x_{3}^{\alpha} \boldsymbol{f}\right) \in W_{-\alpha, x_{3}}^{-1,2}\left(\mathbb{R}_{+}^{3}\right) .
$$

Conversely, let $g \in W_{-\alpha, x_{3}}^{-1,2}\left(\mathbb{R}_{+}^{3}\right)$, and let us consider the mapping

$$
u \mapsto\left(x_{3}^{\alpha-1} u, x_{3}^{\alpha} \nabla u\right),
$$

from $W_{\alpha_{3} x_{3}}^{1,2}\left(\mathbb{R}_{+}^{3}\right)$ into $L^{2}\left(\mathbb{R}_{+}^{3}\right)^{4}$. This mapping is isometric; thus one can consider $W_{\alpha, x_{3}}^{1,2}\left(\mathbb{R}_{+}^{3}\right)$ as a closed sub-space of $L^{2}\left(\mathbb{R}_{+}^{3}\right)^{4}$. Thanks to the theorem of Hahn-Banach, if $g \in W_{-\alpha, x_{3}}^{-1,2}\left(\mathbb{R}_{+}^{3}\right)$, one can extend $g$ to a linear and continuous form on $L^{2}\left(\mathbb{R}_{+}^{3}\right)^{4}$. As a consequence, one deduces from Riesz representation, that there is $\left(f_{0}, \boldsymbol{f}\right) \in L^{2}\left(\mathbb{R}_{+}^{3}\right)^{4}$ such that:

$$
\forall u \in W_{\alpha, x_{3}}^{1,2}\left(\mathbb{R}_{+}^{3}\right), \quad g(u)=\int_{\mathbb{R}_{+}^{3}} f_{0}\left(x_{3}^{\alpha-1} u\right) d x-\int_{\mathbb{R}_{+}^{3}} \boldsymbol{f} \cdot\left(x_{3}^{\alpha} \nabla u\right) d x,
$$

and one deduces that:

$$
g=x_{3}^{\alpha-1} f_{0}+\operatorname{div}\left(x_{3}^{\alpha} \boldsymbol{f}\right) \text { in } \mathbb{R}_{+}^{3} .
$$

According to (13), if $\alpha \neq 1 / 2$ the mapping $u \mapsto\left\|x_{3}^{\alpha} \nabla u\right\|_{L^{2}\left(\mathbb{R}_{+}^{3}\right)^{3}}$ defines a norm on $W_{\alpha, x_{3}}^{1,2}\left(\mathbb{R}_{+}^{3}\right)$ equivalent to $\|\cdot\|_{W_{\alpha, x_{3}}^{1,2}\left(\mathbb{R}_{+}^{3}\right)}$. Then, the above reasoning holds if we replace the mapping $u \mapsto\left(x_{3}^{\alpha-1} u, x_{3}^{\alpha} \nabla u\right)$ by the following one,

$$
u \mapsto x_{3}^{\alpha} \nabla u,
$$

from $W_{\alpha, x_{3}}^{1,2}\left(\mathbb{R}_{+}^{3}\right)$ into $L^{2}\left(\mathbb{R}_{+}^{3}\right)^{3}$, and as expected we can take $f_{0}=0$.

Remark 2.7. Note that for any $\beta \in \mathbb{R}$, the operator:

$$
T_{\beta}: W_{\alpha, x_{3}}^{1,2}\left(\mathbb{R}_{+}^{3}\right) \rightarrow W_{\alpha-\beta, x_{3}}^{1,2}\left(\mathbb{R}_{+}^{3}\right), \quad u \mapsto x_{3}^{\beta} u,
$$

is an isomorphism, and also that the following estimates hold:

$$
\frac{1}{\sqrt{1+\beta^{2}}}\|u\|_{W_{\alpha, x_{3}}^{1,2}\left(\mathbb{R}_{+}^{3}\right)} \leqslant\left\|T_{\beta} u\right\|_{W_{\alpha-\beta, x_{3}}^{1,2}\left(\mathbb{R}_{+}^{3}\right)} \leqslant \sqrt{1+\beta^{2}}\|u\|_{W_{\alpha, x_{3}}^{1,2}\left(\mathbb{R}_{+}^{3}\right)} .
$$

Then, it follows for any $\alpha \in \mathbb{R}$ that $g \in W_{\alpha, x_{3}}^{-1,2}\left(\mathbb{R}_{+}^{3}\right)$ if and only if $x_{3}^{\beta} g \in W_{\alpha-\beta, x_{3}}^{-1,2}\left(\mathbb{R}_{+}^{3}\right)$, with the estimates below:

$$
\frac{1}{\sqrt{1+\beta^{2}}}\|g\|_{W_{\alpha, x_{3}}^{-1,2}\left(\mathbb{R}_{+}^{3}\right)} \leqslant\left\|x_{3}^{\beta} g\right\|_{W_{\alpha-\beta, x_{3}}^{-1,2}\left(\mathbb{R}_{+}^{3}\right)} \leqslant \sqrt{1+\beta^{2}}\|g\|_{W_{\alpha, x_{3}}^{-1,2}\left(\mathbb{R}_{+}^{3}\right)} .
$$


Before finishing this section, we want to compare the space $W_{\alpha, x_{3}}^{1,2}\left(\mathbb{R}_{+}^{3}\right)$ to $\stackrel{\circ}{W}_{\alpha}^{1,2}\left(\mathbb{R}_{+}^{3}\right)$, the closure of $\mathcal{D}\left(\mathbb{R}_{+}^{3}\right)$ in $W_{\alpha}^{1,2}\left(\mathbb{R}_{+}^{3}\right)$.

Firstly, since $\rho^{\beta} \leqslant x_{3}^{\beta}$ for any $\beta \leqslant 0$, we have the following continuous and dense embedding:

$$
\forall \alpha \leqslant 0, \quad W_{\alpha, x_{3}}^{1,2}\left(\mathbb{R}_{+}^{3}\right) \hookrightarrow \stackrel{\circ}{W}_{\alpha}^{1,2}\left(\mathbb{R}_{+}^{3}\right) .
$$

Secondly, when $\alpha \geqslant 0$ the converse situation happens, and we have the dense and continuous embedding below:

$$
\forall \alpha \geqslant 0, \quad \stackrel{\circ}{W}_{\alpha}^{1,2}\left(\mathbb{R}_{+}^{3}\right) \hookrightarrow W_{\alpha, x_{3}}^{1,2}\left(\mathbb{R}_{+}^{3}\right) .
$$

To explain (18), let us first prove the following lemma.

Lemma 2.8. Let $\alpha \in \mathbb{R}$. Then, we have the following inequality:

$$
\forall u \in \stackrel{\circ}{W}_{\alpha}^{1,2}\left(\mathbb{R}_{+}^{3}\right), \quad\left\|\frac{\rho^{\alpha} u}{x_{3}}\right\|_{L^{2}\left(\mathbb{R}_{+}^{3}\right)} \leqslant 2(1+|\alpha|)\|u\|_{W_{\alpha}^{1,2}\left(\mathbb{R}_{+}^{3}\right)} .
$$

Proof. Firstly, we prove (18) for $\alpha=0$. Let $u \in \stackrel{\circ}{W}_{0}^{1,2}\left(\mathbb{R}_{+}^{3}\right)$ and $\left(u_{n}\right) \subset \mathcal{D}\left(\mathbb{R}_{+}^{3}\right)$ a sequence converging to $u$ in $W_{0}^{1,2}\left(\mathbb{R}_{+}^{3}\right)$. From Hardy's inequality (13), the sequence $\left(\frac{u_{n}}{x_{3}}\right)$ is a Cauchy sequence of $L^{2}\left(\mathbb{R}_{+}^{3}\right)$. Therefore, we deduce that $\frac{u}{x_{3}} \in L^{2}\left(\mathbb{R}_{+}^{3}\right)$, with:

$$
\left\|\frac{u}{x_{3}}\right\|_{L^{2}\left(\mathbb{R}_{+}^{3}\right)} \leqslant 2\|\nabla u\|_{L^{2}\left(\mathbb{R}_{+}^{3}\right)^{3}} \leqslant 2\|u\|_{W_{0}^{1,2}\left(\mathbb{R}_{+}^{3}\right)},
$$

that proves (19) when $\alpha=0$. Then, since we already have $\nabla u \in L^{2}\left(\mathbb{R}_{+}^{3}\right)^{3}$, we prove that $u \in W_{0, x_{3}}^{1,2}\left(\mathbb{R}_{+}^{3}\right)$ with:

$$
\|u\|_{W_{0, x_{3}}^{1,2}\left(\mathbb{R}_{+}^{3}\right)} \leqslant \sqrt{5}\|u\|_{W_{0}^{1,2}\left(\mathbb{R}_{+}^{3}\right)},
$$

and (18) is proved for $\alpha=0$. Secondly, we establish (19) for any $\alpha$. Let $u \in \stackrel{\circ}{W}_{\alpha}^{1,2}\left(\mathbb{R}_{+}^{3}\right)$. From (9), the function $\rho^{\alpha} u$ belongs to $\stackrel{\circ}{W}_{0}^{1,2}\left(\mathbb{R}_{+}^{3}\right)$, and hence to $W_{0, x_{3}}^{1,2}\left(\mathbb{R}_{+}^{3}\right)$, as we have just proved it. As a consequence, Hardy's inequality implies that:

$$
\begin{aligned}
\left\|\frac{\rho^{\alpha} u}{x_{3}}\right\|_{L^{2}\left(\mathbb{R}_{+}^{3}\right)} \leqslant 2\left\|\nabla\left(\rho^{\alpha} u\right)\right\|_{L^{2}\left(\mathbb{R}_{+}^{3}\right)^{3}} & \leqslant 2\left(\left\|\rho^{\alpha} \nabla u\right\|_{L^{2}\left(\mathbb{R}_{+}^{3}\right)^{3}}+|\alpha|\left\|\frac{x}{\rho} \rho^{\alpha-1} u\right\|_{L^{2}\left(\mathbb{R}_{+}^{3}\right)}\right) \\
& \leqslant 2(1+|\alpha|)\|u\|_{W_{\alpha}^{1,2}\left(\mathbb{R}_{+}^{3}\right)} .
\end{aligned}
$$

We are now in position to explain (18). Let $\alpha \geqslant 0$ and $u \in \stackrel{\circ}{W}_{\alpha}^{1,2}\left(\mathbb{R}_{+}^{3}\right)$. Then, since $x_{3}^{\alpha} \leqslant \rho^{\alpha}$, we already have $x_{3}^{\alpha} \nabla u \in L^{2}\left(\mathbb{R}_{+}^{3}\right)^{3}$. Besides, if we write $x_{3}^{\alpha-1} u$ in the following way:

$$
x_{3}^{\alpha-1} u=\left(\frac{x_{3}}{\rho}\right)^{\alpha} \frac{\rho^{\alpha} u}{x_{3}},
$$

it follows from Lemma 2.8 that $x_{3}^{\alpha-1} u \in L^{2}\left(\mathbb{R}_{+}^{3}\right)$, and then that $u \in W_{\alpha, x_{3}}^{1,2}\left(\mathbb{R}_{+}^{3}\right)$ with:

$$
\|u\|_{W_{\alpha, x_{3}}^{1,2}\left(\mathbb{R}_{+}^{3}\right)} \leqslant\left(1+4(1+|\alpha|)^{2}\right)\|u\|_{W_{\alpha, x_{3}}^{1,2}\left(\mathbb{R}_{+}^{3}\right)} .
$$

Remark 2.9. These embeddings yield several consequences. Firstly, note that:

$$
W_{0, x_{3}}^{1,2}\left(\mathbb{R}_{+}^{3}\right)=\stackrel{\circ}{W}_{0}^{1,2}\left(\mathbb{R}_{+}^{3}\right),
$$

and also that $\|\cdot\|_{W_{0}^{1,2}\left(\mathbb{R}_{+}^{3}\right)}$ defines an equivalent norm to $\|\cdot\|_{W_{0, x_{3}}^{1,2}\left(\mathbb{R}_{+}^{3}\right)}$ on the space $W_{0, x_{3}}^{1,2}\left(\mathbb{R}_{+}^{3}\right)$. Moreover, $W_{0, x_{3}}^{-1,2}\left(\mathbb{R}_{+}^{3}\right)$ and $W_{0}^{-1,2}\left(\mathbb{R}_{+}^{3}\right)$ are the same distributions. Secondly, observe more generally that:

$$
\begin{array}{ll}
\forall \alpha \leqslant 0 & W_{\alpha, x_{3}}^{-1,2}\left(\mathbb{R}_{+}^{3}\right) \hookrightarrow W_{\alpha}^{-1,2}\left(\mathbb{R}_{+}^{3}\right) ; \\
\forall \alpha \geqslant 0, & W_{\alpha}^{-1,2}\left(\mathbb{R}_{+}^{3}\right) \hookrightarrow W_{\alpha, x_{3}}^{-1,2}\left(\mathbb{R}_{+}^{3}\right) .
\end{array}
$$




\section{Weak solutions}

Let us start off with a first situation. Here, we seek weak solutions to Problem (1) in the space $W_{0, x_{3}}^{1,2}\left(\mathbb{R}_{+}^{3}\right)$. For convenience, let us write Problem (1) in the following way:

$$
\left\{\begin{aligned}
-\Delta u+\frac{1}{x_{3}} \frac{\partial u}{\partial x_{3}} & =f & & \text { in } \mathbb{R}_{+}^{3} \\
u & =0 & & \text { on } \mathbb{R}^{2}
\end{aligned}\right.
$$

where $f=x_{3} g$. Then, we have the following proposition.

Proposition 3.1. Let $f \in W_{0, x_{3}}^{-1,2}\left(\mathbb{R}_{+}^{3}\right)$. Then, Problem (21) has a unique solution $u$ in the space $W_{0, x_{3}}^{1,2}\left(\mathbb{R}_{+}^{3}\right)$ and satisfying the estimate:

$$
\|u\|_{W_{0, x_{3}}^{1,2}\left(\mathbb{R}_{+}^{3}\right)} \leqslant 2\|f\|_{W_{0, x_{3}}^{-1,2}\left(\mathbb{R}_{+}^{3}\right)} .
$$

Proof. We observe by Proposition 2.6 that $u \in W_{0, x_{3}}^{1,2}\left(\mathbb{R}_{+}^{3}\right)$ is a solution to (21) if and only if $u$ satisfies the formulation below:

$$
\left\{\begin{array}{l}
\forall v \in W_{0, x_{3}}^{1,2}\left(\mathbb{R}_{+}^{3}\right) \\
\int_{\mathbb{R}_{+}^{3}} \nabla u \cdot \nabla v d x+\int_{\mathbb{R}_{+}^{3}} \frac{\partial u}{\partial x_{3}} \frac{v}{x_{3}} d x=\langle f, v\rangle .
\end{array}\right.
$$

Here the duality is taken in the sense of $W_{0, x_{3}}^{-1,2}\left(\mathbb{R}_{+}^{3}\right), W_{0, x_{3}}^{1,2}\left(\mathbb{R}_{+}^{3}\right)$. Next, let us focus on the bilinear form

$$
u, v \in W_{0, x_{3}}^{1,2}\left(\mathbb{R}_{+}^{3}\right), \quad(u, v) \mapsto \int_{\mathbb{R}_{+}^{3}} \nabla u \cdot \nabla v d x+\int_{\mathbb{R}_{+}^{3}} \frac{v}{x_{3}} \frac{\partial u}{\partial x_{3}} d x
$$

It is clearly continuous and coercive on $W_{0, x_{3}}^{1,2}\left(\mathbb{R}_{+}^{3}\right)$, since one has for any $u \in \mathcal{D}\left(\mathbb{R}_{+}^{3}\right)$ and then by density for $u \in W_{0, x_{3}}^{1,2}\left(\mathbb{R}_{+}^{3}\right)$ :

$$
\int_{\mathbb{R}_{+}^{3}} \frac{u}{x_{3}} \frac{\partial u}{\partial x_{3}} d x=\frac{1}{2}\left\|\frac{u}{x_{3}}\right\|_{L^{2}\left(\mathbb{R}_{+}^{3}\right)}^{2} .
$$

Then Proposition 3.1 follows as a consequence of Lax-Milgram's lemma.

We can also prove the existence and uniqueness of a range of weak solutions, living in the more general space $W_{\alpha, x_{3}}^{1,2}\left(\mathbb{R}_{+}^{3}\right)$. However, we need to consider particular values of the parameter $\alpha$. Note that the following result includes Proposition 3.1.

Theorem 3.2. Let $-3 / 2<\alpha<1 / 2$ and $g \in W_{\alpha+1, x_{3}}^{-1,2}\left(\mathbb{R}_{+}^{3}\right)$. Then, there exists a unique $u \in W_{\alpha, x_{3}}^{1,2}\left(\mathbb{R}_{+}^{3}\right)$ solution to Problem (1). Besides, for some positive constant $C_{\alpha}\left({ }^{2}\right)$ depending only on $\alpha, u$ satisfies the estimate below:

$$
\|u\|_{W_{\alpha, x_{3}}^{1,2}\left(\mathbb{R}_{+}^{3}\right)} \leqslant C_{\alpha}\|g\|_{W_{\alpha+1, x_{3}}^{-1,2}\left(\mathbb{R}_{+}^{3}\right)} .
$$

More precisely, $C_{\alpha}$ is defined by:

$$
C_{\alpha}=\frac{\left(5-4 \alpha+4 \alpha^{2}\right) \sqrt{1+(1+2 \alpha)^{2}}}{4(\alpha-3 / 2)(1 / 2-\alpha)} .
$$

Proof. To enlighten the proof, we set $E=W_{\alpha, x_{3}}^{1,2}\left(\mathbb{R}_{+}^{3}\right)$ and $F=W_{-(\alpha+1), x_{3}}^{1,2}\left(\mathbb{R}_{+}^{3}\right)$, and we denote by $F^{\prime}$ the dual space to $F$. Then, let us consider the linear operator:

$$
A: u \in E \mapsto-\operatorname{div}\left(\frac{\nabla u}{x_{3}}\right) \in F^{\prime} .
$$

\footnotetext{
${ }^{2}$ Until the end of the paper, $C_{\alpha}$ denotes any positive constant depending only on $\alpha$.
} 
Notice that according to Proposition 2.6, $A$ is clearly defined and continuous. Then, let us also consider the continuous bilinear form:

$$
(u, v) \in E \times F, \quad a(u, v)=\langle A u, v\rangle_{F^{\prime}, F} .
$$

Firstly, it is clear that $u \in E$ is a solution to Problem (1) if and only if $u$ satisfies the following variational formulation:

$$
\forall v \in F, \quad a(u, v)=\langle g, v\rangle_{F^{\prime}, F} .
$$

Secondly, we prove the existence and uniqueness of $u \in E$ satisfying (26), by applying Babǔska-Brezzki's theorem ( $c f$. Babǔska [4], $c f$. Brezzi [10]). Therefore, we need to establish the inf-sup condition below:

$$
\inf _{u \in E-\{0\}} \sup _{v \in F-\{0\}} \frac{|a(u, v)|}{\|u\|_{E}\|v\|_{F}} \geqslant \frac{1}{C_{\alpha}}>0 .
$$

Let $u \in E-\{0\}$. Then, since $F=T_{2 \alpha+1} E$ see (14), we deduce from (15) that:

$$
\sup _{v \in F-\{0\}} \frac{a(u, v)}{\|u\|_{E}\|v\|_{F}} \geqslant \frac{a\left(u, x_{3}^{2 \alpha+1} u\right)}{\sqrt{1+(1+2 \alpha)^{2}}\|u\|_{E}^{2}} .
$$

Then, observe the following computations, obtained first for $u \in \mathcal{D}\left(\mathbb{R}_{+}^{3}\right)$ and then by density for $u \in E$, see Theorem 2.3:

$$
\begin{aligned}
a\left(u, x_{3}^{2 \alpha+1} u\right) & =\int_{\mathbb{R}_{+}^{3}} x_{3}^{\alpha} \nabla u \cdot x_{3}^{-(\alpha+1)} \nabla\left(x_{3}^{2 \alpha+1} u\right) d x \\
& =\left\|x_{3}^{\alpha} \nabla u\right\|_{L^{2}\left(\mathbb{R}_{+}^{3}\right)}^{2 \alpha}+\frac{2 \alpha+1}{2} \int_{\mathbb{R}_{+}^{3}} x_{3}^{2 \alpha-1} \frac{\partial u^{2}}{\partial x_{3}} d x \\
& =\left\|x_{3}^{\alpha} \nabla u\right\|_{L^{2}\left(\mathbb{R}_{+}^{3}\right)}^{2}+\beta\left\|x_{3}^{\alpha-1} u\right\|_{L^{2}\left(\mathbb{R}_{+}^{3}\right)}^{2},
\end{aligned}
$$

where $\beta=\frac{1-4 \alpha^{2}}{2}$. Next, we have for any $\left.\left.\varepsilon \in\right] 0,1\right]$ :

$$
a\left(u, x_{3}^{2 \alpha+1} u\right)=\varepsilon\left\|x_{3}^{\alpha} \nabla u\right\|_{L^{2}\left(\mathbb{R}_{+}^{3}\right)}^{2}+(1-\varepsilon)\left\|x_{3}^{\alpha} \nabla u\right\|_{L^{2}\left(\mathbb{R}_{+}^{3}\right)}^{2}+\beta\left\|x_{3}^{\alpha-1} u\right\|_{L^{2}\left(\mathbb{R}_{+}^{3}\right)}^{2},
$$

and Hardy's inequality (13) yields that:

$$
a\left(u, x_{3}^{2 \alpha+1} u\right) \geqslant \varepsilon\left\|x_{3}^{\alpha} \nabla u\right\|_{L^{2}\left(\mathbb{R}_{+}^{3}\right)}^{2}+\left[(1-\varepsilon) \frac{(2 \alpha-1)^{2}}{4}+\beta\right]\left\|x_{3}^{\alpha-1} u\right\|_{L^{2}\left(\mathbb{R}_{+}^{3}\right)}^{2} .
$$

Then, for $\varepsilon=\frac{4(\alpha-3 / 2)(1 / 2-\alpha)}{5-4 \alpha+4 \alpha^{2}}$, belonging to ]0, 1] as soon as $\alpha$ belongs to $]-3 / 2,1 / 2[$, note that the constants in (28) are the same. Thus we get:

$$
a\left(u, x_{3}^{2 \alpha+1} u\right) \geqslant \frac{4(\alpha-3 / 2)(1 / 2-\alpha)}{5-4 \alpha+4 \alpha^{2}}\|u\|_{E}^{2}
$$

and

$$
\frac{1}{C_{\alpha}}=\frac{4(\alpha-3 / 2)(1 / 2-\alpha)}{\left(5-4 \alpha+4 \alpha^{2}\right) \sqrt{1+(1+2 \alpha)^{2}}} .
$$

As a conculsion, we have proved that for any $-3 / 2<\alpha<\frac{1}{2}$, there is a positive constant $C_{\alpha}$, depending only on $\alpha$, such that:

$$
a\left(u, x_{3}^{2 \alpha+1} u\right) \geqslant \frac{1}{C_{\alpha}}\|u\|_{E}^{2},
$$

implying (27), and ending the proof. 
Remark 3.3. If $\alpha \leqslant-3 / 2$ or $\alpha \geqslant 1 / 2$ and $g \in W_{\alpha+1, x_{3}}^{-1,2}\left(\mathbb{R}_{+}^{3}\right)$, existence and uniqueness of solutions to (1) is still an open question.

In the second part of this section, we establish existence and uniqueness of weak solutions to Problem (1) in the space $W_{\alpha}^{1,2}\left(\mathbb{R}_{+}^{3}\right)$ see (4). For convenience, we shall consider the equivalent problem (21).

Theorem 3.4. Let $-\frac{1+\sqrt{2}}{2} \leqslant \alpha<\frac{3-\sqrt{6}}{2}$ and let $f \in W_{\alpha}^{-1,2}\left(\mathbb{R}_{+}^{3}\right)$. Then, there is a unique $u \in \stackrel{\circ}{W}_{\alpha}^{1,2}\left(\mathbb{R}_{+}^{3}\right)$ solution to Problem $(21)$, and there is a constant $C_{\alpha}>0$ depending only on $\alpha$, such that $u$ satisfies:

$$
\|u\|_{W_{\alpha}^{1,2}\left(\mathbb{R}_{+}^{3}\right)} \leqslant C_{\alpha}\|f\|_{W_{\alpha}^{-1,2}\left(\mathbb{R}_{+}^{3}\right)} \cdot
$$

More precisely, $C_{\alpha}$ is given by (33) if $0 \leqslant \alpha<\frac{3-\sqrt{6}}{2}$, and by (34) if $-\frac{1+\sqrt{2}}{2}<\alpha<0$. Proof. The proof is similar to the one of Theorem 3.2. For convenience, we set $E=\stackrel{\circ}{W}_{\alpha}^{1,2}\left(\mathbb{R}_{+}^{3}\right)$ and $F=\stackrel{\circ}{W}_{-\alpha}^{1,2}\left(\mathbb{R}_{+}^{3}\right)$, and we denote by $F^{\prime}$ the dual space to $F$. We consider the linear operator:

$$
B: u \in E \mapsto-\Delta u+\frac{1}{x_{3}} \frac{\partial u}{\partial x_{3}} \in F^{\prime}
$$

clearly defined and continuous, according to Lemma 2.8. Then, we also consider the continuous bilinear form:

$$
(u, v) \in E \times F, \quad b(u, v)=\langle B u, v\rangle_{F^{\prime}, F} .
$$

Firstly, it is clear that $u \in E$ is a solution to Problem (21) if and only if $u$ satisfies the following variational formulation:

$$
\forall v \in F, \quad b(u, v)=\langle f, v\rangle_{F^{\prime}, F} .
$$

Then, we prove existence and uniqueness of $u \in E$ satisfying (30), by using BabǔskaBrezzki's theorem. Let $u \in E-\{0\}$. Then, since $F=T_{\rho, 2 \alpha} E$ see (9), we deduce from (10) that:

$$
\sup _{v \in F-\{0\}} \frac{b(u, v)}{\|u\|_{E}\|v\|_{F}} \geqslant \frac{b\left(u, \rho^{2 \alpha} u\right)}{\sqrt{1+4 \alpha^{2}}\|u\|_{E}^{2}} .
$$

For any $u \in \mathcal{D}\left(\mathbb{R}_{+}^{3}\right)$ observe that:

$$
b\left(u, \rho^{2 \alpha} u\right)=\int_{\mathbb{R}_{+}^{3}} \nabla u \cdot \nabla\left(\rho^{2 \alpha} u\right) d x+\int_{\mathbb{R}_{+}^{3}}\left(\rho^{\alpha} \frac{u}{x_{3}}\right)\left(\rho^{\alpha} \frac{\partial u}{\partial x_{3}}\right) d x .
$$

Next, thanks to the formulas below:

$$
\Delta\left(\rho^{2 \alpha}\right)=6 \alpha \rho^{2 \alpha-2}+4 \alpha(\alpha-1)|x|^{2} \rho^{2 \alpha-4}, \quad \frac{\partial}{\partial x_{3}}\left(\frac{\rho^{2 \alpha}}{x_{3}}\right)=-\frac{\rho^{2 \alpha}}{x_{3}^{2}}+2 \alpha \rho^{2 \alpha-2},
$$

one deduces that:

$$
\begin{aligned}
& \int_{\mathbb{R}_{+}^{3}} \nabla u \cdot \nabla\left(\rho^{2 \alpha} u\right) d x \\
= & \left\|\rho^{\alpha} \nabla u\right\|_{L^{2}\left(\mathbb{R}_{+}^{3}\right)^{3}}^{2}-\frac{1}{2} \int_{\mathbb{R}_{+}^{3}} \Delta\left(\rho^{2 \alpha}\right) u^{2} d x \\
= & \left\|\rho^{\alpha} \nabla u\right\|_{L^{2}\left(\mathbb{R}_{+}^{3}\right)^{3}}^{2}+\int_{\mathbb{R}_{+}^{3}}\left[-3 \alpha-2 \alpha(\alpha-1) \frac{|x|^{2}}{\rho^{2}}\right] \rho^{2(\alpha-1)} u^{2} d x,
\end{aligned}
$$


and also that:

$$
\begin{aligned}
\int_{\mathbb{R}_{+}^{3}} \rho^{2 \alpha} \frac{u}{x_{3}} \frac{\partial u}{\partial x_{3}} d x & =-\frac{1}{2} \int_{\mathbb{R}_{+}^{3}} \frac{\partial}{\partial x_{3}}\left(\frac{\rho^{2 \alpha}}{x_{3}}\right) u^{2} d x=\frac{1}{2} \int_{\mathbb{R}_{+}^{3}}\left(\frac{\rho^{2 \alpha}}{x_{3}^{2}}-2 \alpha \rho^{2 \alpha-2}\right) u^{2} d x \\
& =\int_{\mathbb{R}_{+}^{3}}\left(\frac{1}{2} \frac{\rho^{2}}{x_{3}^{2}}-\alpha\right) \rho^{2(\alpha-1)} u^{2} d x \\
& \geqslant \int_{\mathbb{R}_{+}^{3}}\left(\frac{1}{2}-\alpha\right) \rho^{2(\alpha-1)} u^{2} d x
\end{aligned}
$$

By density, it follows that for any $u \in E$ :

$$
b\left(u, \rho^{2 \alpha} u\right) \geqslant\left\|\rho^{\alpha} \nabla u\right\|_{L^{2}\left(\mathbb{R}_{+}^{3}\right)^{3}}^{2}+\int_{\mathbb{R}_{+}^{3}} \beta(x) \rho^{2(\alpha-1)} u^{2} d x,
$$

where $\beta$ is defined by:

$$
x \in \mathbb{R}_{+}^{3}, \quad \beta(x)=-4 \alpha+\frac{1}{2}+2 \alpha(1-\alpha)\left|\frac{x}{\rho}\right|^{2} .
$$

Several situation appear with respect to $\alpha$.

First case 1. If $\alpha \in[0,1]$, then $\beta(x) \geqslant-4 \alpha+\frac{1}{2}$ and Hardy's inequality (8) yields for $\varepsilon \in] 0,1]$ :

$$
\begin{aligned}
b\left(u, \rho^{2 \alpha} u\right) & \geqslant \varepsilon\left\|\rho^{\alpha} \nabla u\right\|_{L^{2}\left(\mathbb{R}_{+}^{3}\right)^{3}}^{2}+\int_{\mathbb{R}_{+}^{3}}\left[\frac{(2 \alpha+1)^{2}(1-\varepsilon)}{4}+\beta(x)\right] \rho^{2(\alpha-1)} u^{2} d x \\
& \geqslant \varepsilon\left\|\rho^{\alpha} \nabla u\right\|_{L^{2}\left(\mathbb{R}_{+}^{3}\right)^{3}}^{2}+\int_{\mathbb{R}_{+}^{3}}\left[\frac{(2 \alpha+1)^{2}(1-\varepsilon)}{4}+\frac{1}{2}-4 \alpha\right] \rho^{2(\alpha-1)} u^{2} d x .
\end{aligned}
$$

Then, we chose $\varepsilon$ such that:

$$
\varepsilon=\frac{(2 \alpha+1)^{2}(1-\varepsilon)}{4}+\frac{1}{2}-4 \alpha,
$$

leading to:

$$
\varepsilon=\frac{4\left(\alpha-\frac{3+\sqrt{6}}{2}\right)\left(\alpha-\frac{3-\sqrt{6}}{2}\right)}{5+4 \alpha+4 \alpha^{2}},
$$

and hence belonging to $] 0,1]$ as soon as $\alpha \in\left[0, \frac{3-\sqrt{6}}{2}[\right.$. Therefore, one has:

$$
b\left(u, \rho^{2 \alpha} u\right) \geqslant \frac{4\left(\alpha-\frac{3+\sqrt{6}}{2}\right)\left(\alpha-\frac{3-\sqrt{6}}{2}\right)}{5+4 \alpha+4 \alpha^{2}}\|u\|_{E}^{2},
$$

and $C_{\alpha}$ is given by:

$$
\frac{1}{C_{\alpha}}=\frac{4\left(\alpha-\frac{3+\sqrt{6}}{2}\right)\left(\alpha-\frac{3-\sqrt{6}}{2}\right)}{\left(5+4 \alpha+4 \alpha^{2}\right) \sqrt{1+4 \alpha^{2}}}
$$

Second case. If $\alpha<0$ or $\alpha>1$, then:

$$
\beta(x) \geqslant-4 \alpha+\frac{1}{2}+2 \alpha(1-\alpha)=-2\left(\alpha+\frac{1+\sqrt{2}}{2}\right)\left(\alpha+\frac{1-\sqrt{2}}{2}\right)
$$

and $1>\beta(x) \geqslant 0$ as soon as $\alpha \in\left[-\frac{1+\sqrt{2}}{2}, 0[\right.$. As a consequence, one has:

$$
b\left(u, \rho^{2 \alpha} u\right) \geqslant-2\left(\alpha+\frac{1+\sqrt{2}}{2}\right)\left(\alpha+\frac{1-\sqrt{2}}{2}\right)\|u\|_{E}^{2},
$$


and:

$$
\frac{1}{C_{\alpha}}=\frac{2\left(\alpha+\frac{1+\sqrt{2}}{2}\right)\left(\alpha+\frac{1-\sqrt{2}}{2}\right)}{\sqrt{1+4 \alpha^{2}}} .
$$

As a conculsion, we have proved that for any $-\frac{1+\sqrt{2}}{2} \leqslant \alpha<\frac{3-\sqrt{6}}{2}$, there is a positive constant $C_{\alpha}$, depending only on $\alpha$, such that:

$$
b\left(u, \rho^{2 \alpha} u\right) \geqslant \frac{1}{C_{\alpha}}\|u\|_{E}^{2},
$$

implying an analogous inf-sup condition (27) for $b$ instead of $a$, and ending the proof.

Remark 3.5. Firstly, observe that the case $\alpha=0$ was already treated in Proposition 3.1 , according to $(20)$.

Secondly, for any $\alpha<-\frac{1+\sqrt{2}}{2}$ or $\alpha \geqslant \frac{3-\sqrt{6}}{2}$ and $f \in W_{\alpha}^{-1,2}\left(\mathbb{R}_{+}^{3}\right)$, the existence of weak solutions to (21) is an open problem.

\section{Strong solutions}

Let $-3 / 2<\alpha<1 / 2, g \in W_{\alpha+1, x_{3}}^{-1,2}\left(\mathbb{R}_{+}^{3}\right)$ and consider the solution $u \in W_{\alpha, x_{3}}^{1,2}\left(\mathbb{R}_{+}^{3}\right)$ to Problem (1) given by Theorem 3.2. We readily see that $u$ satisfies:

$$
-x_{3}^{\alpha+1} \Delta u+x_{3}^{\alpha} \frac{\partial u}{\partial x_{3}}=x_{3}^{\alpha+2} g .
$$

Since we already have $x_{3}^{\alpha} \frac{\partial u}{\partial x_{3}} \in L^{2}\left(\mathbb{R}_{+}^{3}\right)$, we deduce that:

$$
x_{3}^{\alpha+2} g \in L^{2}\left(\mathbb{R}_{+}^{3}\right) \Rightarrow x_{3}^{\alpha+1} \Delta u \in L^{2}\left(\mathbb{R}_{+}^{3}\right) .
$$

Thus, by taking such a distribution $g$, we expect more regularity on any second order derivative $D^{2} u$ of $u$, and more precisely that $x_{3}^{\alpha+1} D^{2} u \in L^{2}\left(\mathbb{R}_{+}^{3}\right)$.

Theorem 4.1. Let $-3 / 2<\alpha<1 / 2$ and $g \in \mathcal{D}^{\prime}\left(\mathbb{R}_{+}^{3}\right)$ such that $x_{3}^{\alpha+2} g \in L^{2}\left(\mathbb{R}_{+}^{3}\right)$. Then $g \in W_{\alpha+1, x_{3}}^{-1,2}\left(\mathbb{R}_{+}^{3}\right)$ and the unique solution $u \in W_{\alpha, x_{3}}^{1,2}\left(\mathbb{R}_{+}^{3}\right)$ given by Theorem 3.2 satisfies:

$$
x_{3}^{\alpha+1} D^{2} u \in L^{2}\left(\mathbb{R}_{+}^{3}\right) .
$$

Moreover, there is a constant $C_{\alpha}>0$, depending only on $\alpha$, such that:

$$
\left\|x_{3}^{\alpha+1} D^{2} u\right\|_{L^{2}\left(\mathbb{R}_{+}^{3}\right)} \leqslant C_{\alpha}\left\|x_{3}^{\alpha+2} g\right\|_{L^{2}\left(\mathbb{R}_{+}^{3}\right)} .
$$

Proof. Let us assume that $x_{3}^{\alpha+2} g \in L^{2}\left(\mathbb{R}_{+}^{3}\right)$. By Proposition 2.6, observe that $g \in$ $W_{\alpha+1, x_{3}}^{-1,2}\left(\mathbb{R}_{+}^{3}\right)$, with:

$$
\|g\|_{W_{\alpha+1, x_{3}}^{-1,2}\left(\mathbb{R}_{+}^{3}\right)} \leqslant\left\|x_{3}^{\alpha+2} g\right\|_{L^{2}\left(\mathbb{R}_{+}^{3}\right)} .
$$

Thus, thanks to Theorem 3.2, there is a unique $u \in W_{\alpha, x_{3}}^{1,2}\left(\mathbb{R}_{+}^{3}\right)$ solution to (1) and a constant $C_{\alpha}>0$, depending on $\alpha$, such that:

$$
\|u\|_{W_{\alpha, x_{3}}^{1,2}\left(\mathbb{R}_{+}^{3}\right)} \leqslant C_{\alpha}\|g\|_{W_{\alpha+1, x_{3}}^{-1,2}\left(\mathbb{R}_{+}^{3}\right)} \leqslant C_{\alpha}\left\|x_{3}^{\alpha+2} g\right\|_{L^{2}\left(\mathbb{R}_{+}^{3}\right)} .
$$

Now, if we set $v=x_{3}^{\alpha+1} u$, it follows from (35) that:

$$
\Delta v=x_{3}^{\alpha+2} g+\alpha(\alpha+1) x_{3}^{\alpha-1} u+(2 \alpha+3) x_{3}^{\alpha} \frac{\partial u}{\partial x_{3}},
$$


implying finally $\Delta v \in L^{2}\left(\mathbb{R}_{+}^{3}\right)$. Therefore, one deduces from Corollary 3.4 of [2], that there is a unique $w \in W_{0}^{2,2}\left(\mathbb{R}_{+}^{3}\right)\left({ }^{3}\right)$ such that:

$$
\Delta w=\Delta v \text { in } \mathbb{R}_{+}^{3}, \quad w=0 \text { on } \Gamma,
$$

and, according to (38), there is a constant $C_{\alpha}>0$ satisfying:

$$
\|w\|_{W_{0}^{2,2}\left(\mathbb{R}_{+}^{3}\right)} \leqslant C_{\alpha}\|\Delta v\|_{L^{2}\left(\mathbb{R}_{+}^{3}\right)} \leqslant C_{\alpha}\left\|x_{3}^{\alpha+2} g\right\|_{L^{2}\left(\mathbb{R}_{+}^{3}\right)} .
$$

Moreover, from (14) one has $v \in W_{-1, x_{3}}^{1,2}\left(\mathbb{R}_{+}^{3}\right)$ and then $v \in W_{-1}^{1,2}\left(\mathbb{R}_{+}^{3}\right)$, see (17). Thus, $z=v-w$ belongs to $W_{-1}^{1,2}\left(\mathbb{R}_{+}^{3}\right)$ and satisfies:

$$
\Delta z=0 \text { in } \mathbb{R}_{+}^{3}, \quad z=0 \text { on } \Gamma,
$$

which implies that $z=0$ (see [2] Theorem 3.2). As a consequence, $v=w$ belongs to $W_{0}^{2,2}\left(\mathbb{R}_{+}^{3}\right)$, and in particular one has for $i, j=1,2,3$ and from (39):

$$
\begin{gathered}
\frac{\partial^{2}}{\partial x_{i} \partial x_{j}}\left(x_{3}^{\alpha+1} u\right) \in L^{2}\left(\mathbb{R}_{+}^{3}\right), \\
\left\|\frac{\partial^{2}}{\partial x_{i} \partial x_{j}}\left(x_{3}^{\alpha+1} u\right)\right\|_{L^{2}\left(\mathbb{R}_{+}^{3}\right)} \leqslant C_{\alpha}\left\|x_{3}^{\alpha+2} g\right\|_{L^{2}\left(\mathbb{R}_{+}^{3}\right)} .
\end{gathered}
$$

Then, since $u \in W_{\alpha, x_{3}}^{1,2}\left(\mathbb{R}_{+}^{3}\right)$, one deduces from (40) that:

$$
x_{3}^{\alpha+1} \frac{\partial^{2} u}{\partial x_{i} \partial x_{j}} \in L^{2}\left(\mathbb{R}_{+}^{3}\right),
$$

and the estimate (37) follows from (41) and (38).

In Theorem 4.1, we have studied a first situation where the weak solution of Theorem 3.2 is in fact a strong solution. We give in the sequel another case of figure, leading to the same conclusion: instead of considering a datum $g$ such that $x_{3}^{\alpha+2} g \in$ $L^{2}\left(\mathbb{R}_{+}^{3}\right)$, we assume now that $x_{3}^{\alpha+1} g \in L^{2}\left(\mathbb{R}_{+}^{3}\right)$ in addition to $g \in W_{\alpha+1, x_{3}}^{-1,2}\left(\mathbb{R}_{+}^{3}\right)$. Therefore we need to use another method, based on differential quotients technique (cf. Nirenberg [14]), for which we recall now the basics.

Notation 4.2. For any $\eta \in \mathbb{R}^{2} \times\{0\}$ with $\eta \neq 0$, and any measurable function $u$ defined on $\mathbb{R}_{+}^{3}$, we set:

$$
x \in \mathbb{R}_{+}^{3}, \quad D_{\eta} u(x)=\frac{u(x+\eta)-u(x)}{|\eta|} .
$$

Recall that for any $u, v \in L^{2}\left(\mathbb{R}_{+}^{3}\right)$, one has the following relation:

$$
\int_{\mathbb{R}_{+}^{3}} u D_{\eta} v d x=\int_{\mathbb{R}_{+}^{3}} v D_{-\eta} u d x
$$

and that if $u \in H^{1}\left(\mathbb{R}_{+}^{3}\right)$, the following estimate holds:

$$
\left\|D_{\eta} u\right\|_{L^{2}\left(\mathbb{R}_{+}^{3}\right)} \leqslant\left\|\nabla^{\prime} u\right\|_{L^{2}\left(\mathbb{R}_{+}^{3}\right)^{2}} .
$$

We also recall the following essential result, whose proof is classical.

${ }^{3}$ Here, $W_{0}^{2,2}\left(\mathbb{R}_{+}^{3}\right)$ denotes the set of functions $u \in W_{-1}^{1,2}\left(\mathbb{R}_{+}^{3}\right)$ with second order derivates $D^{2} u \in$ $L^{2}\left(\mathbb{R}_{+}^{3}\right)^{9}$. This space is endowed with the norm:

$$
\|u\|_{W_{0}^{2,2}\left(\mathbb{R}_{+}^{3}\right)}=\left(\|u\|_{W_{-1}^{1,2}\left(\mathbb{R}_{+}^{3}\right)}^{2}+\left\|D^{2} u\right\|_{L^{2}\left(\mathbb{R}_{+}^{3}\right)^{9}}^{2}\right)^{1 / 2} .
$$


Proposition 4.3. Let $u \in L^{2}\left(\mathbb{R}_{+}^{3}\right)$ such that

$$
\forall \eta \in \mathbb{R}^{2} \times\{0\} \text { with } \eta \neq 0, \quad\left\|D_{\eta} u\right\|_{L^{2}\left(\mathbb{R}_{+}^{3}\right)} \leqslant C,
$$

where $C>0$ is a constant not depending on $\eta$. Then

$$
\nabla^{\prime} u \in L^{2}\left(\mathbb{R}_{+}^{3}\right)^{2} \quad \text { and } \quad\left\|\nabla^{\prime} u\right\|_{L^{2}\left(\mathbb{R}_{+}^{3}\right)^{2}} \leqslant C \sqrt{2} .
$$

We give a last preliminary result.

Proposition 4.4. Let $\alpha \in \mathbb{R}$, and $g \in \mathcal{D}^{\prime}\left(\mathbb{R}_{+}^{3}\right)$ such that $x_{3}^{\alpha} g \in L^{2}\left(\mathbb{R}_{+}^{3}\right)$. Then, $g \in L_{\mathrm{loc}}^{1}\left(\mathbb{R}_{+}^{3}\right)$ and we have the following assertions.

i) For any $\eta \in \mathbb{R}^{2} \times\{0\}$ with $\eta \neq 0$, the function $D_{\eta} g$ belongs to $W_{\alpha, x_{3}}^{-1,2}\left(\mathbb{R}_{+}^{3}\right)$ and the following estimate holds:

$$
\left\|D_{\eta} g\right\|_{W_{\alpha, x_{3}\left(\mathbb{R}_{+}^{3}\right)}^{-1,2}} \leqslant\left\|x_{3}^{\alpha} g\right\|_{L^{2}\left(\mathbb{R}_{+}^{3}\right)} .
$$

ii) For any $v \in W_{-\alpha, x_{3}}^{1,2}\left(\mathbb{R}_{+}^{3}\right)$, one has $D_{\eta} v \in W_{-\alpha, x_{3}}^{1,2}\left(\mathbb{R}_{+}^{3}\right)$, and if in addition $g \in$ $W_{\alpha, x_{3}}^{-1,2}\left(\mathbb{R}_{+}^{3}\right)$, we have according to claim i):

$$
\forall v \in W_{-\alpha, x_{3}}^{1,2}\left(\mathbb{R}_{+}^{3}\right), \quad\left\langle D_{\eta} g, v\right\rangle=\left\langle g, D_{-\eta} v\right\rangle .
$$

Proof.

i) Since $x_{3}^{\alpha} g \in L^{2}\left(\mathbb{R}_{+}^{3}\right)$ and since $x_{3}^{\alpha} D_{\eta} g=D_{\eta}\left(x_{3}^{\alpha} g\right)$ one has $x_{3}^{\alpha} D_{\eta} g \in L^{2}\left(\mathbb{R}_{+}^{3}\right)$. Then, one deduces for any $\varphi \in \mathcal{D}\left(\mathbb{R}_{+}^{3}\right)$ that:

$$
\begin{aligned}
\left|\int_{\mathbb{R}_{+}^{3}} \varphi x_{3}^{\alpha} D_{\eta} g d x\right|=\left|\int_{\mathbb{R}_{+}^{3}} x_{3}^{\alpha} g D_{-\eta} \varphi d x\right| & \leqslant\left\|x_{3}^{\alpha} g\right\|_{L^{2}\left(\mathbb{R}_{+}^{3}\right)}\left\|D_{-\eta} \varphi\right\|_{L^{2}\left(\mathbb{R}_{+}^{3}\right)} \\
& \leqslant\left\|x_{3}^{\alpha} g\right\|_{L^{2}\left(\mathbb{R}_{+}^{3}\right)}\|\varphi\|_{W_{0, x_{3}}^{1,2}\left(\mathbb{R}_{+}^{3}\right)} .
\end{aligned}
$$

As a consequence, $x_{3}^{\alpha} D_{\eta} g \in W_{0, x_{3}}^{-1,2}\left(\mathbb{R}_{+}^{3}\right)$ and then $D_{\eta} g \in W_{\alpha, x_{3}}^{-1,2}\left(\mathbb{R}_{+}^{3}\right)$, see Remark 2.7 .

ii) For any $v \in W_{-\alpha, x_{3}}^{1,2}\left(\mathbb{R}_{+}^{3}\right)$ one has $D_{\eta} v \in W_{-\alpha, x_{3}}^{1,2}\left(\mathbb{R}_{+}^{3}\right)$, and observe that:

$$
x_{3}^{-\alpha-1} D_{\eta} v=D_{\eta}\left(x_{3}^{-\alpha-1} v\right), \quad x_{3}^{-\alpha} \nabla\left(D_{\eta} v\right)=D_{\eta}\left(x_{3}^{-\alpha} \nabla v\right) .
$$

Then, let assume in addition that $g \in W_{\alpha, x_{3}}^{-1,2}\left(\mathbb{R}_{+}^{3}\right)$. Let $v \in W_{-\alpha, x_{3}}^{1,2}\left(\mathbb{R}_{+}^{3}\right)$ and $\left(v_{k}\right)_{k} \subset$ $\mathcal{D}\left(\mathbb{R}_{+}^{3}\right)$ a sequence converging to $v$ in $W_{-\alpha, x_{3}}^{1,2}\left(\mathbb{R}_{+}^{3}\right)$. One obtains:

$$
\begin{aligned}
\left\langle D_{\eta} g, v\right\rangle=\lim _{k \rightarrow+\infty}\left\langle D_{\eta} g, v_{k}\right\rangle & =\lim _{k \rightarrow+\infty} \int_{\mathbb{R}_{+}^{3}} v_{k} D_{\eta} g d x \\
& =\lim _{k \rightarrow+\infty} \int_{\mathbb{R}_{+}^{3}} g D_{-\eta} v_{k} d x,
\end{aligned}
$$

and relation (45) holds, since $g \in W_{\alpha, x_{3}}^{-1,2}\left(\mathbb{R}_{+}^{3}\right)$ and $D_{-\eta} v_{k}$ converges to $D_{-\eta} v$ in $W_{-\alpha, x_{3}}^{1,2}\left(\mathbb{R}_{+}^{3}\right)$.

We are now in position to prove the following theorem.

Theorem 4.5. Assume that $-3 / 2<\alpha<1 / 2$ and let $g \in W_{\alpha+1, x_{3}}^{-1,2}\left(\mathbb{R}_{+}^{3}\right)$. If moreover $x_{3}^{\alpha+1} g \in L^{2}\left(\mathbb{R}_{+}^{3}\right)$, the unique solution $u \in W_{\alpha, x_{3}}^{1,2}\left(\mathbb{R}_{+}^{3}\right)$ to Problem (1) satisfies additionally:

$$
x_{3}^{\alpha-1} \nabla^{\prime} u \in L^{2}\left(\mathbb{R}_{+}^{3}\right)^{2}, \quad x_{3}^{\alpha+1} \nabla\left(\frac{1}{x_{3}} \nabla u\right) \in L^{2}\left(\mathbb{R}_{+}^{3}\right)^{9} .
$$

Moreover, there is a constant $C_{\alpha}>0$, depending only on $\alpha$, such that:

$$
\left\|x_{3}^{\alpha-1} \nabla^{\prime} u\right\|_{L^{2}\left(\mathbb{R}_{+}^{3}\right)^{2}}+\left\|x_{3}^{\alpha+1} \nabla\left(\frac{1}{x_{3}} \nabla u\right)\right\|_{L^{2}\left(\mathbb{R}_{+}^{3}\right)^{9}} \leqslant C_{\alpha}\left\|x_{3}^{\alpha+1} g\right\|_{L^{2}\left(\mathbb{R}_{+}^{3}\right)} .
$$


Proof. Since $g \in W_{\alpha+1, x_{3}}^{-1,2}\left(\mathbb{R}_{+}^{3}\right)$, let us consider the unique weak solution $u \in W_{\alpha, x_{3}}^{1,2}\left(\mathbb{R}_{+}^{3}\right)$ to Problem (21), see Theorem 3.2. We recall that $u$ satisfies (26), and in particular one has:

$$
\forall v \in W_{-(\alpha+1), x_{3}}^{1,2}\left(\mathbb{R}_{+}^{3}\right), \quad \int_{\mathbb{R}_{+}^{3}} x_{3}^{\alpha} \nabla u \cdot x_{3}^{-(\alpha+1)} \nabla v d x=\langle g, v\rangle,
$$

where the duality is taken in the sense $W_{\alpha+1, x_{3}}^{-1,2}\left(\mathbb{R}_{+}^{3}\right), W_{-(\alpha+1), x_{3}}^{1,2}\left(\mathbb{R}_{+}^{3}\right)$. By (14) and Proposition 4.4, we can consider as a test function in $(48) v_{\eta}=D_{-\eta} D_{\eta}\left(x_{3}^{2 \alpha+1} u\right)$, for any $\eta \in \mathbb{R}^{2} \times\{0\}$ with $\eta \neq 0$. On the one hand, one proves by relation (42) that:

$$
\int_{\mathbb{R}_{+}^{3}} x_{3}^{\alpha} \nabla u \cdot x_{3}^{-(\alpha+1)} \nabla v_{\eta} d x=a\left(D_{\eta} u, x_{3}^{2 \alpha+1} D_{\eta} u\right),
$$

with $D_{\eta} u \in W_{\alpha, x_{3}}^{1,2}\left(\mathbb{R}_{+}^{3}\right)$. Then, the inf-sup condition (27) implies:

$$
\int_{\mathbb{R}_{+}^{3}} x_{3}^{\alpha} \nabla u \cdot x_{3}^{-(\alpha+1)} \nabla v_{\eta} d x \geqslant C_{\alpha}\left\|D_{\eta} u\right\|_{W_{\alpha, x_{3}}^{1,2}\left(\mathbb{R}_{+}^{3}\right)}^{2},
$$

for some positive constant $C_{\alpha}$, depending only on $\alpha$. On the other hand, observe by Proposition 4.4, and by (15) that:

$$
\begin{aligned}
\left|\left\langle g, D_{-\eta} D_{\eta} x_{3}^{2 \alpha+1} u\right\rangle\right| & =\left|\left\langle D_{\eta} g, D_{\eta} x_{3}^{2 \alpha+1} u\right\rangle\right| \\
& \leqslant\left\|x_{3}^{\alpha+1} g\right\|_{L^{2}\left(\mathbb{R}_{+}^{3}\right)}\left\|x_{3}^{2 \alpha+1} D_{\eta} u\right\|_{W_{-(\alpha+1), x_{3}}^{1,2}\left(\mathbb{R}_{+}^{3}\right)} \\
& \leqslant C_{\alpha}\left\|x_{3}^{\alpha+1} g\right\|_{L^{2}\left(\mathbb{R}_{+}^{3}\right)}\left\|D_{\eta} u\right\|_{W_{\alpha, x_{3}}^{1,2}\left(\mathbb{R}_{+}^{3}\right)} .
\end{aligned}
$$

Collecting the previous estimates, we finally deduce that:

$$
\left\|D_{\eta} u\right\|_{W_{\alpha, x_{3}}^{1,2}\left(\mathbb{R}_{+}^{3}\right)} \leqslant C_{\alpha}\left\|x_{3}^{\alpha+1} g\right\|_{L^{2}\left(\mathbb{R}_{+}^{3}\right)},
$$

Consequently, Proposition 4.3 ensures that for $i=1,2$ :

$$
x_{3}^{\alpha-1} \frac{\partial u}{\partial x_{i}} \in L^{2}\left(\mathbb{R}_{+}^{3}\right), \quad x_{3}^{\alpha} \nabla\left(\frac{\partial u}{\partial x_{i}}\right) \in L^{2}\left(\mathbb{R}_{+}^{3}\right)^{3},
$$

with the expected estimates. Then, if we write equation (1) in the following way:

$$
x_{3}^{\alpha+1} \frac{\partial}{\partial x_{3}}\left(\frac{1}{x_{3}} \frac{\partial u}{\partial x_{3}}\right)=-\sum_{i=1,2} x_{3}^{\alpha} \frac{\partial^{2} u}{\partial x_{i}^{2}}-x_{3}^{\alpha} g,
$$

we deduce from (50) that:

$$
x_{3}^{\alpha+1} \frac{\partial}{\partial x_{3}}\left(\frac{1}{x_{3}} \frac{\partial u}{\partial x_{3}}\right) \in L^{2}\left(\mathbb{R}_{+}^{3}\right)
$$

and (46) follows.

To finish this section, let us make the following observation. We consider $|\alpha|<1 / 2$, $g \in W_{\alpha+1, x_{3}}^{-1,2}\left(\mathbb{R}_{+}^{3}\right)$ such that $x_{3}^{\alpha+1} g \in L^{2}\left(\mathbb{R}_{+}^{3}\right)$, and the solution $u \in W_{\alpha, x_{3}}^{1,2}\left(\mathbb{R}_{+}^{3}\right)$ to $(1)$. Since $x_{3}^{\alpha+1} g \in L^{2}\left(\mathbb{R}_{+}^{3}\right)$, then $g \in W_{\alpha, x_{3}}^{-1,2}\left(\mathbb{R}_{+}^{3}\right)$ and we deduce firstly from Theorem 3.2 that $u \in W_{\alpha-1, x_{3}}^{1,2}\left(\mathbb{R}_{+}^{3}\right)$, and secondly from Theorem 4.1 that $x_{3}^{\alpha} D^{2} u \in L^{2}\left(\mathbb{R}_{+}^{3}\right)$. As a consequence, we can state the following corollary.

Corollary 4.6. If $|\alpha|<1 / 2, g \in W_{\alpha+1, x_{3}}^{-1,2}\left(\mathbb{R}_{+}^{3}\right)$ and $x_{3}^{\alpha+1} g \in L^{2}\left(\mathbb{R}_{+}^{3}\right)$, the solution $u \in W_{\alpha, x_{3}}^{1,2}\left(\mathbb{R}_{+}^{3}\right)$ to Problem (1) also belongs to $W_{\alpha-1, x_{3}}^{1,2}\left(\mathbb{R}_{+}^{3}\right)$ and satisfies additionally:

$$
x_{3}^{\alpha} D^{2} u \in L^{2}\left(\mathbb{R}_{+}^{3}\right)^{9} .
$$

Besides, there is a constant $C_{\alpha}>0$ depending only on $\alpha$ such that:

$$
\|u\|_{W_{\alpha-1, x_{3}}^{1,2}\left(\mathbb{R}_{+}^{3}\right)}+\left\|x_{3}^{\alpha} D^{2} u\right\|_{L^{2}\left(\mathbb{R}_{+}^{3}\right)^{9}} \leqslant C_{\alpha}\left\|x_{3}^{\alpha+1} g\right\|_{L^{2}\left(\mathbb{R}_{+}^{3}\right)} .
$$


Remark 4.7. According to Theorem 4.1 and the above corollary, an optimal space of strong solutions to Problem (1) is in fact:

$$
W_{\alpha, x_{3}}^{2,2}\left(\mathbb{R}_{+}^{3}\right)=\left\{u \in \mathcal{D}^{\prime}\left(\mathbb{R}_{+}^{3}\right) / x_{3}^{\alpha+|k|-2} D^{|k|} u \in L^{2}\left(\mathbb{R}_{+}^{3}\right): 0 \leqslant|k| \leqslant 2\right\},
$$

endowed with the norm:

$$
\|u\|_{W_{\alpha, x_{3}}^{2,2}\left(\mathbb{R}_{+}^{3}\right)}=\left(\sum_{0 \leqslant|k| \leqslant 2}\left\|x_{3}^{\alpha+|k|-2} D^{|k|} u\right\|_{L^{2}\left(\mathbb{R}_{+}^{3}\right)}^{2}\right)^{1 / 2} .
$$

We leave the study of this space for further works.

Let us finish the comparaison, by saying that when $-3 / 2<\alpha \leqslant-1 / 2$, Theorem 4.5 may be seen as a generalization of Theorem 4.1.

Let us enforce this point with the following example. Let a distribution $g$ such that $\sqrt{x_{3}} g \in L^{2}\left(\mathbb{R}_{+}^{3}\right)$, which implies to choose formally $\alpha=-3 / 2$ in the assumptions of Theorem 4.1. Unfortunately, such a distribution does not satisfy the statement of Theorem 3.2, and since we are not able for the moment to establish existence and uniqueness of a weak solution to (1), we add the extra assumption $\sqrt{\overline{x_{3}}} g \in W_{0, x_{3}}^{-1,2}\left(\mathbb{R}_{+}^{3}\right)$ of Theorem 4.5.

\section{References}

[1] C. Amrouche, F. Dahoumane, R. Luce, and G. Vallet. On the hydrostatic Stokes approximation with non homogeneous boundary conditions. Differential Equations and Applications, 2(3):419-446, 2010.

[2] C. Amrouche and S. Nečasová. Laplace equation in the half space with a non homogeneous Dirichlet boundary condition. Mathematica Bohemica, 126(2):265$274,2001$.

[3] P. Azérad and F. Guillén-González. Mathematical justification of the hydrostatic approximation in the primitive equations of geophysical fluid dynamics. SIAM J. Math. Anal., 33(4):847-859, 2001.

[4] Ivo Babuška. The finite element method with Lagrangian multipliers. Numer. Math., 20:179-192, 1972/73.

[5] O. Besson and M. R. Laydi. Some estimates for the anisotropic Navier-Stokes and for the Hydrostatic approximation. M2AN, 26(27):855-965, 1992.

[6] T.Z. Boulmezaoud. Espaces de sobolev avec poids pour l'équation de laplace dans le demi-espace. C. R. Acad. Sci. Paris Série. 1, 328:221-226, 1999.

[7] D. Bresch, F. Guillén-González, and J. Lemoine. A note on a degenerate elliptic equation with applications for lakes and seas. Electron. J. Differential Equations, 2004(42):1-13, 2004.

[8] D. Bresch, J. Lemoine, and J. Simon. A geostrophic model with vertical diffusion. Nonlinear Analysis, Theory, Methods and Applications, 43(4):449-470, 2001.

[9] D. Bresch and M. Sy. Porous convection in rotating thin domains: the planetary geostrophic equations, used in geophysical fluid dynamics, revisited. Cont. Mech. Thermodyn., 15(3):247-263, 2003. 
[10] F. Brezzi. On the existence, uniqueness and approximation of saddle-point problems arising from Lagrangian multipliers. Rev. Française Automat. Informat. Recherche Opérationnelle Sér. Rouge, 8(R-2):129-151, 1974.

[11] T. Chacón Rebollo and F. Guillén González. An intrinsic analysis of existence of solutions for the hydrostatic approximation of Navier-Stokes equations. C. R. Acad. Sci. Paris Série. 1, 330:841-846, 2000.

[12] P. Grisvard. Espaces intermédiaires entre espaces de Sobolev avec poids. Ann. Scuola Norm. Sup. Pisa (3), 17(3):255-296, 1963.

[13] B. Hanouzet. Espaces de sobolev avec poids. Rend. del. Sem. Mat. della Univ. di Padova, XLVI:227-272, 1971.

[14] L. Nirenberg. On elliptic partial differential equations. Ann. Scuola Norm. Sup. Pisa (3), 13:115-162, 1959.

[15] Roger Temam and Mohammed Ziane. Some mathematical problems in geophysical fluid dynamics. In Handbook of mathematical fluid dynamics. Vol. III, pages 535-657. North-Holland, Amsterdam, 2004. 